\title{
O USO DA CLASSIFICAÇÃO FUNCIONAL DO FITOPLÂNCTON COMO INSTRUMENTO DE MONITORAMENTO AMBIENTAL
}

\author{
Renata Martins dos Santos ${ }^{1}$
}

Raquel Aparecida Moreira ${ }^{2}$

\section{Lidiane Cristina da Silva ${ }^{3}$}

Resumo: Algas fitoplanctônicas são importantes na avaliação da qualidade da água, refletindo a dinâmica do ecossistema. O objetivo principal deste estudo foi avaliar a riqueza, abundância e a distribuição dos grupos funcionais da comunidade fitoplanctônica nas águas do Parque do Bicão na cidade de São Carlos, São Paulo. Amostras de água para análise das variáveis físicas e químicas e do fitoplâncton foram coletadas nos meses de abril e agosto de 2013 em três pontos. A comunidade fitoplanctônica foi analisada através da sua densidade, dos grupos funcionais e dos índices biológicos. A análise da comunidade fitoplanctônica do Parque do Bicão revelou uma riqueza expressiva de espécies, foram identificadas 30 espécies distribuídas em sete classes taxonômicas. As clorofíceas, cianobactérias e diatomáceas foram componentes importantes desta comunidade. As maiores riqueza e densidade foram registradas na amostragem de abril de 2013. Em ambas as amostragens nos pontos P2 (lago) e P3 (lançamento de esgoto) foram registradas as maiores diversidades. A classificação do fitoplâncton em grupos funcionais evidenciou a ocorrência de 12 associações. Entre estas, o grupo funcional $\mathrm{J}$,

\footnotetext{
${ }^{1}$ Bióloga, Programa de Pós-Gradução em Ecologia e Recursos Naturais, Universidade Federal de São Carlos. renata cordis@yahoo.com.br

${ }^{2}$ Bióloga, Programa de Pós-Gradução em Ecologia e Recursos Naturais, Universidade Federal de São Carlos. raquel.moreira88@hotmail.com

${ }^{3}$ Bióloga, Programa de Pós-Gradução em Ecologia e Recursos Naturais, Universidade Federal de São Carlos. lidianecris2004@yahoo.com.br
} 
representado principalmente por Desmodesmus quadricauda (Turpin) Brébrisson, refletiu claramente as condições mais eutróficas dos pontos P2 e P3. A abordagem dos grupos funcionais do fitoplâncton constituiu uma ferramenta útil para a compreensão da comunidade fitoplanctônica neste sistema urbano. Conclui-se que a região onde se localiza o Parque do Bicão, deve ser considerada para a utilização na preservação do equilíbrio natural e proteção das comunidades aquáticas. Contudo, isso só será possível quando as medidas que visem a sua recuperação e preservação forem efetivamente implantadas.

Palavras-chave: Grupos funcionais. Algas. Eutrofização.

\section{INTRODUÇÃO}

Os estudos de avaliação da qualidade da água que incluam análise qualitativa e quantitativa dos organismos planctônicos são importantes, pois estes refletem a dinâmica do ecossistema (TUNDISI, 2003). Segundo Margalef (1983), a comunidade planctônica funciona como "sensor refinado das propriedades ambientais".

O manejo inadequado dos corpos d'água geralmente acelera o processo da eutrofização, ou seja, o enriquecimento de um corpo d'água por nutrientes orgânicos e minerais, levando à proliferação das comunidades biológicas e à baixa qualidade da água utilizada para diversos fins. Esse processo é um problema recorrente e comum em todo o mundo, e como conseqüência, ocorre a proliferação de algas, causada pela oferta excessiva de nutrientes (CROSSETI; BICUDO, 2005).

As águas, que passam pelo processo de eutrofização, podem apresentar desequilíbrio de elementos e nutrientes, diminuição do teor de oxigênio dissolvido, alteração do $\mathrm{pH}$ e proliferação de diversos organismos nocivos à saúde humana. Como resultado da falta de manejo adequado desses ambientes, observa-se o crescimento acelerado dessas comunidades onde predominam as espécies tóxicas de cianofíceas (TUCCl et al., 2006).

Na tentativa de propor modelos e padrões para comunidade fitoplanctônica em em ambientes de água doce que apresentam diferentes níveis de trofia, pesquisadores desenvolveram hipóteses para uma classificação mais abrangente (HUTCHINSON, 1961; 
REYNOLDS, 1988). Um modelo foi proposto por Reynolds et al. (2002) no qual as espécies do fitoplâncton foram agrupadas em 31 grupos funcionais, denominados por códigos alfanuméricos, baseando-se em suas estratégias de sobrevivência, tolerâncias e sensibilidades. Estes eram definidos pelos atributos fisiológicos, morfológicos e ecológicos de espécies que potencial e alternativamente podem dominar ou co-dominar um sistema (HUSZAR et al., 2003). Estes grupos são frequentemente polifiléticos e respondem similarmente a um determinado conjunto de condições ambientais, possuindo características adaptativas para sobrevivência e dominância em distintos ambientes (REYNOLS et al., 2002).

A classificação do fitoplâncton de acordo com grupos funcionais despertou o interesse da comunidade científica, pois este tipo de abordagem contribui para a análise da distribuição e dinâmica das populações fitoplanctônicas naturais (REYNOLDS et al. 2002; REYNOLDS, 2006), é mais eficiente no biomonitoramento ambiental do que agrupamentos filogenéticos (HUSZAR \& CARACO, 1998), tem se tornado fundamental para uma melhor compreensão e descrição das comunidades e é uma forma viável de predizer respostas e avaliar as mudanças nos ecossistemas aquáticos causados pelas ações antrópicas. Além disso, considera a tolerância ou a sensibilidade das espécies às variáveis físicas e químicas do sistema aquático onde são encontrados (CUNHA \& CALIJURI, 2011), permitindo a avaliação da influência das condições hidráulicas de um reservatório (por exemplo, o tempo de residência), das características morfométricas e de uso e ocupação da bacia hidrográfica sobre as algas (BORGES et al. 2008).

Portanto, o uso de grupos funcionais do fitoplâncton para avaliar as modificações ocorridas em um sistema tem provado ser muito útil e eficiente. Assim, o objetivo deste estudo foi avaliar a riqueza, abundância e a distribuição dos grupos funcionais da comunidade fitoplanctônica nas águas do Parque do Bicão na cidade de São Carlos.

\section{ÁREA DE ESTUDO}

O Parque Municipal "Veraldo Sbampato" conhecido popularmente como Parque do

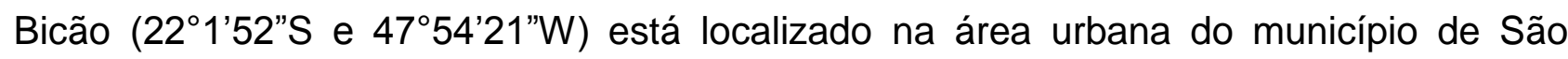
Carlos. Ele se localiza no local da nascente do rio Medeiros, que apresenta um uso e ocupação do solo excessiva nas cabeceiras, tornando inviável sua recuperação integral, 
além da presença de lançamento clandestino de esgoto e problemas de criminalidade há vários anos (TARPANI, 2008).

No interior do parque existem três nascentes que, no passado, sofreram sérias intervenções antrópicas devido à urbanização. Estas nascentes formam um curso d'água que atravessa todo o parque e apresenta sinais de assoreamento. Constata-se também na área forte presença de esgotos domésticos clandestinos, lançados à jusante das nascentes, no córrego do Medeiros, ainda dentro do Parque do Bicão (Figura 1).

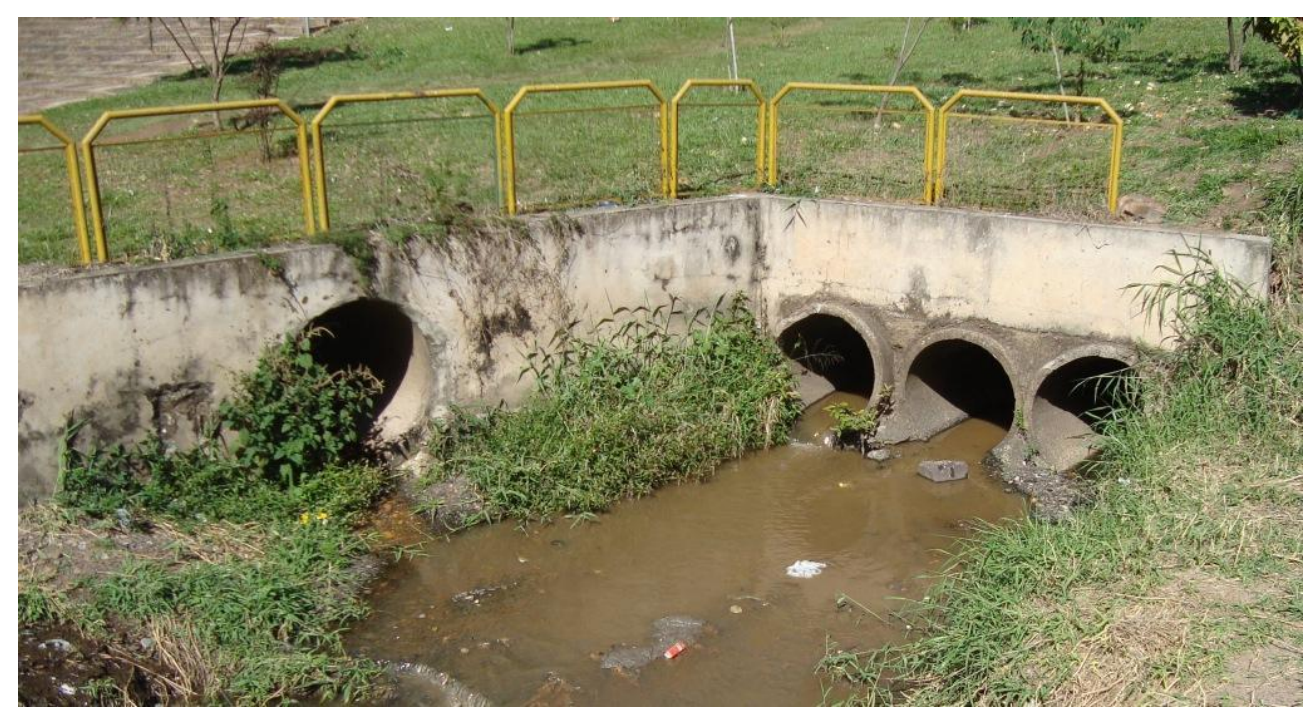

Figura 1. Aspecto geral do lançamento de esgoto no curso d'água que atravessa o Parque do Bicão.

\section{MATERIAIS E MÉTODOS}

\section{Variáveis abióticas}

A coleta foi realizada em abril e agosto de 2013 em três pontos localizados no

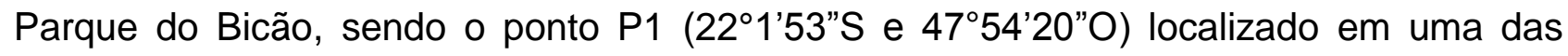
nascentes do rio Medeiros, o ponto P2 (22 $1^{\prime} 53^{\prime \prime S}$ e $\left.47^{\circ} 54^{\prime} 21^{\prime \prime O}\right)$ no lago central do parque e o ponto P3 (22 $1^{\prime} 51^{\prime \prime S}$ e $47^{\circ} 54^{\prime} 26^{\prime \prime}$ ) à jusante do local de lançamento de esgoto.

A temperatura da água, $\mathrm{pH}$, condutividade elétrica e oxigênio dissolvido foram mensurados in situ com uma sonda portátil da marca Horiba ${ }^{\circledR}$, modelo U-10. 


\section{Variáveis bióticas}

As amostras para as análises quantitativas do fitoplâncton foram coletadas de forma direta na superfície em frascos de polietileno e preservadas com solução 1\% de lugol acético. Para a identificação utilizou-se bibliografia especializada utilizando-se o sistema de classificação adotado por Bicudo e Menezes (2005).

A densidade populacional do fitoplâncton foi estimada com o auxílio de um microscópio invertido pelo método da sedimentação de Utermöhl (1958), para o qual foram utilizadas câmaras de sedimentação de volumes entre 2,5 e $20 \mathrm{~mL}$, dependendo da concentração de algas e de material em suspensão em cada amostra. O tempo de sedimentação foi de pelo menos três horas para cada centímetro de altura da câmara (Margalef, 1983). Os organismos (células, colônias e filamentos) foram quantificados em campos aleatórios (Uhelingher, 1964), em aumento de 400x, sendo enumerados pelo menos 100 indivíduos da espécie mais freqüente. O cálculo da densidade fitoplanctônica (org $\mathrm{mL}^{-1}$ ) foi efetuado conforme descrito em Ros (1979)

Com os dados, calculou-se a densidade e a abundância das espécies. A definição dos grupos funcionais foi efetuada com base em Reynolds et al. (2002). Os índices de diversidade, dominância e equitabilidade foram calculados utilizando-se o programa estatístico PAST version 1.94 (HAMMER et al., 2001). O índice de constância de Dajóz (ICD) foi calculado para cada espécie. Quando ICD $\geq 70$ as espécies são consideradas constantes; quando $30 \leq I C D<70$ representa as espécies freqüentes, quando $10 \leq I C D<$ 30 representa as espécies esporádicas e ICD < 10 representa as espécies raras (DAJOZ, 1978).

\section{RESULTADOS E DISCUSSÃO}

Os valores das características físicas e químicas da água nos pontos de coleta no Parque do Bicão estão apresentados na Tabela 1. 
Tabela 1: Características físicas e químicas da água nos pontos de coleta no Parque do Bicão nos meses de abril e agosto de 2013.

\begin{tabular}{|c|c|c|c|c|c|c|}
\hline & \multicolumn{3}{|c|}{ Abril } & \multicolumn{3}{|c|}{ Agosto } \\
\hline & P1 & P2 & P3 & P1 & P2 & P3 \\
\hline Temperatura da água $\left({ }^{\circ} \mathrm{C}\right)$ & 23,0 & 23,1 & 23,5 & 21,8 & 20,7 & 21,3 \\
\hline $\mathrm{pH}$ & 6,38 & 6,57 & 6,76 & 6,21 & 6,85 & 6,98 \\
\hline Oxigênio dissolvido (mg L $\left.{ }^{-1}\right)$ & 4,45 & 9,37 & 6,98 & 7,52 & 9,88 & 8,88 \\
\hline Condutividade elétrica $\left(\mu \mathrm{S} \mathrm{cm}^{-1}\right)$ & 127 & 92,0 & 96,0 & 181 & 143 & 169 \\
\hline
\end{tabular}

A temperatura da água foi relativamente maior na coleta realizada em abril de 2013, como esperado para o período de coleta caracterizado como um período seco. $\mathrm{O}$ pH manteve-se ácido com valores menores que 7,0. A concentração de oxigênio dissolvido registrada está dentro da faixa de ambientes aquáticos urbanos no Brasil (GENTIL et al., 2008). Os valores da condutividade elétrica na coleta realizada em agosto de 2013 foram superiores àqueles registrados em abril de $2013 \mathrm{em}$ todos os pontos.

Os parâmetros físicos e químicos analisados mostraram que os pontos de coleta no Parque do Bicão são pouco profundos, com águas ácidas devido à presença de grande quantidade de ácidos húmicos o que também colaborou para os expressivos valores da condutividade elétrica. Valores altos desta variável foi registrado por Gentil et al. (2008) estudando a dinâmica do fitoplâncton em um algo urbano em São Paulo.

A determinação dos fatores que podem influenciar a distribuição da comunidade fitoplanctônica, é necessária para o entendimento da dinâmica deste grupo de organismos. Os fatores abióticos podem interferir na densidade das populações planctônicas alterando as interações bióticas dentro do sistema (ESPÍNDOLA, 1994). Diversos estudos têm demonstrado a influência de fatores ambientais como condições climáticas, $\mathrm{pH}$, condutividade, temperatura, oxigênio dissolvido e disponibilidade de nutrientes sobre os organismos fitoplanctônicos (REYNOLDS, 1984; DANTAS et al., 2009; LONGHI; BEISNER, 2009).

O conjunto de espécies da comunidade fitoplanctônica reflete a interação das características de um ecossistema, em certo período (REYNOLDS, 1984). No presente estudo foram identificadas 30 espécies da comunidade fitoplanctônica (Tabela 2). As classes Cyanophyceae e Bacillariophyceae foram representadas pelo maior número de 
espécies (nove cada), seguidas por Chlorophyceae (sete espécies). Euglenophyceae foi representada por três espécies e Zygnematophyceae e Cryptophyeceae por apenas uma espécie. Essa maior abundância em termos de espécies das classes Cyanophyceae, Chlorophyceae e Bacillariophyceae é um resultado frequentemente observado em corpos de água do Brasil (GENTIL et al., 2008; SANTOS, 2010).

Em relação a riqueza de espécies entre os pontos, esta foi maior nos pontos P3 e P2 (13 e 12 espécies respectivamente) na coleta realizada em abril de 2013. A menor riqueza foi registrada no ponto $\mathrm{P} 1$ em ambas as amostragens (uma espécie em abril e duas espécies em agosto).

A espécie mais frequente (67\%) no presente estudo foi Desmodesmus quadricauda (Turpin) Brébrisson, registrada nos pontos P2 e P3 em ambas as amostragens. Várias espécies foram registradas em apenas uma coleta sendo: Aphanocapsa koordersi Strom, Merismopedia glauca (Ehrenberg) Kützing, Planktolyngbya limnetica (Lemmermann) Komárková-Legnerová \& Cronberg, Rhabdoderma lineare Schmidle \& Lauterborn, Chlorella vulgaris Kessler \& Huss, Dictyosphaerium pulchellum Wood, Pediastrum duplex Meyen, Euglena sp. Ehrenberg, Lepocinclis sp. Perti, Cyclotella sp. (Kützing) Brébisson, Nitzschia sp. Tabellaria sp. Kützing registradas apenas em abril de 2013 e Eutetramorus fotti (Hind.) Kom., Desmidium sp. (C.A. Agardh), Phacus sp. Pochmann, Urosolenia cf. longiseta (Zacharias) Edlund et Stoermer e Cryptomonas sp. Ehrenberg apenas em agosto de 2013. 
Tabela 2: Lista das espécies fitoplanctônicas registradas nos pontos de coleta no Parque do Bicão nos meses de abril e agosto de 2013.

\begin{tabular}{|c|c|c|c|c|c|c|c|c|}
\hline & \multicolumn{3}{|c|}{ Abril } & \multicolumn{3}{|c|}{ Agosto } & \multirow{2}{*}{$\%$} & \multirow{2}{*}{ ICD } \\
\hline & $\mathbf{P 1}$ & P2 & P3 & P1 & $\mathbf{P 2}$ & P3 & & \\
\hline \multicolumn{9}{|l|}{ Cyanophyceae } \\
\hline Aphanocapsa delicatissima W. West et G.S. West & & $x$ & & & $\mathrm{x}$ & & 33 & Frequente \\
\hline Aphanocapsa koordersii Strom & & $x$ & & & & & 17 & Esporádica \\
\hline Epigloeosphaera brasilica Azevedo et al & & & $x$ & & & & 17 & Esporádica \\
\hline Limnothrix sp. (Woloszynska) Merffert & & & $x$ & & $x$ & & 33 & Frequente \\
\hline Merismopedia glauca (Ehrenberg) & & & $x$ & & & & 17 & Esporádica \\
\hline $\begin{array}{l}\text { Planktolyngbya limnetuca (Lemmermann) } \\
\text { Komárková-Legnerová \& Cronberg }\end{array}$ & & $\mathrm{x}$ & & & & & 17 & Esporádica \\
\hline Pseudanabaena galeata Böcher & $x$ & & $x$ & & & & 33 & Frequente \\
\hline Raphidiopsis curvata F.E.Fritsch \& M.F.Rich & & $x$ & & & $\mathrm{x}$ & & 33 & Frequente \\
\hline Rhabdoderma lineare Schmidle \& Lauterborn & & & $x$ & & & & 17 & Esporádica \\
\hline \multicolumn{9}{|l|}{ Chlorophyceae } \\
\hline Chlorella vulgaris Kessler \& Huss & & $x$ & & & & & 17 & Esporádica \\
\hline Desmodesmus quadricauda (Turpin) Brébrisson & & $x$ & $x$ & & $\mathrm{x}$ & $x$ & 67 & Frequente \\
\hline Dictyosphaerium pulchellum Wood & & & $x$ & & & & 17 & Esporádica \\
\hline Eutetramorus fotti (Hind.) Kom. & & & & & & $x$ & 17 & Esporádica \\
\hline Oocystis sp. Lemmermann & & & & $x$ & & $x$ & 33 & Frequente \\
\hline Pediastrum duplex Meyen & & & $x$ & & & & 17 & Esporádica \\
\hline Scenedesmus acuminatus (Lagerheim) Chodat & & $x$ & $x$ & & & & 33 & Frequente \\
\hline \multicolumn{9}{|l|}{ Zygnematophyceae } \\
\hline Desmidium sp. (C.A. Agardh) & & & & & & $x$ & 17 & Esporádica \\
\hline \multicolumn{9}{|l|}{ Euglenophycea } \\
\hline Euglena sp. Ehrenberg & & & $x$ & & & & 17 & Esporádica \\
\hline Lepocinclis sp. Perti & & $x$ & & & & & 17 & Esporádica \\
\hline Phacus sp. Pochmann & & & & & & $x$ & 17 & Esporádica \\
\hline \multicolumn{9}{|l|}{ Bacillariophyceae } \\
\hline Aulacoseira granulata (Ehrenberg) Simonsen & & & $x$ & & $x$ & & 33 & Frequente \\
\hline Cyclotella sp. (Kützing) Brébisson & & $x$ & & & & & 17 & Esporádica \\
\hline Fragilaria sp. Lyngbye & & $x$ & $x$ & & $x$ & $x$ & 67 & Frequente \\
\hline Navicula sp. Bory de Saint-Vincent & & & & $x$ & & $x$ & 33 & Frequente \\
\hline Nitzschia sp. & & & $x$ & & & & 17 & Esporádica \\
\hline Pinnularia sp. Ehrenberg & & & & & $x$ & $x$ & 33 & Frequente \\
\hline Surirella sp. Turpin & & & & & $x$ & $x$ & 33 & Frequente \\
\hline Tabellaria sp. Kützing & & $x$ & & & & & 17 & Esporádica \\
\hline $\begin{array}{l}\text { Urosolenia cf. longiseta (Zacharias) Edlund et } \\
\text { Stoermer }\end{array}$ & & & & & $x$ & & 17 & Esporádica \\
\hline \multicolumn{9}{|l|}{ Cryptophyceae } \\
\hline Cryptomonas sp. Ehrenberg & & $x$ & & & $x$ & & 33 & Frequente \\
\hline Total riqueza & 1 & 12 & 13 & 2 & 10 & 9 & & \\
\hline
\end{tabular}


Os resultados da abundância relativa das classes fitoplanctônicas demonstram dominância de Chlorophyceae em todos os pontos amostrados com exceção no ponto P1 em abril de 2013 (Figura 2). Bacillariophyceae foi a segunda mais representativa em termos numéricos.

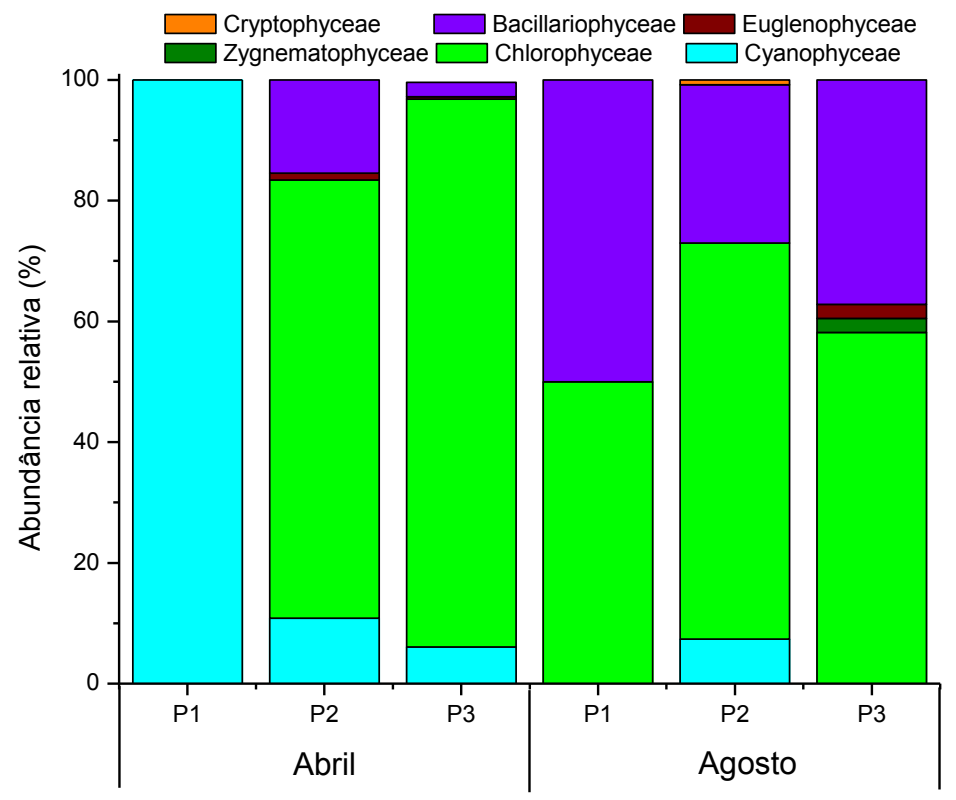

Figura 2. Abundâcia relativa (\%) das classes fitoplanctônicas nos pontos de coleta no Parque do Bicão nos meses de abril e agosto de 2013.

A densidade numérica do fitoplâncton está representada na Tabela 3 e Figura 3. A maior densidade total foi registrada no ponto P3 $\left(8.951 \mathrm{org} \mathrm{mL}^{-1}\right.$ ) em abril de 2013 e a menor no ponto P1 (7 org $\mathrm{mL}^{-1}$ ) em agosto de 2013. Entre as coletas verifica-se que em abril de 2013 foram registrados os maiores valores de densidade para os pontos P1 e P3.

A espécie $D$. quadricauda foi expressivamente representativa em ambos os períodos de coleta sendo registrada em elevadas densidades nos pontos P2 e P3. Na amostragem em abril esta foi maior no ponto $\mathrm{P} 3\left(8.013 \mathrm{org} \mathrm{mL}^{-1}\right)$ e na amostragem em agosto no ponto $\mathrm{P} 2$ (2.888 org $\left.\mathrm{mL}^{-1}\right)$. O ponto $\mathrm{P} 3$ está localizado em uma região próxima a um lançamento de esgoto, sendo provavelmente rico em nutrientes. No ponto P2, localizado no lago central do parque, as condições não são diferentes, uma vez que neste local a presença de patos favorece também o enriquecimento por nutrientes. 
Tabela 3: Densidade (org $\mathrm{mL}^{-1}$ ) das espécies fitoplanctônicas registradas nos pontos de coleta no Parque do Bicão nos meses de abril e agosto de 2013.

\begin{tabular}{|c|c|c|c|c|c|c|}
\hline & \multicolumn{3}{|c|}{ Abril } & \multicolumn{3}{|c|}{ Agosto } \\
\hline & P1 & $\mathbf{P 2}$ & P3 & P1 & $\mathbf{P 2}$ & P3 \\
\hline \multicolumn{7}{|l|}{ Cyanophyceae } \\
\hline Aphanocapsa delicatissima W. West et G.S. West & & 18 & & & 72 & \\
\hline Aphanocapsa koordersii Strom & & 18 & & & & \\
\hline Epigloeosphaera brasilica Azevedo et al & & & 144 & & & \\
\hline Limnothrix sp. (Woloszynska) Merffert & & & 144 & & 36 & \\
\hline Merismopedia glauca (Ehrenberg) & & & 36 & & & \\
\hline $\begin{array}{l}\text { Planktolyngbya limnetuca (Lemmermann) } \\
\text { Komárková-Legnerová \& Cronberg }\end{array}$ & & 289 & 0 & & & \\
\hline Pseudanabaena galeata Böcher & 14 & & 144 & & & \\
\hline Raphidiopsis curvata F.E.Fritsch \& M.F.Rich & & 18 & & & 217 & \\
\hline Rhabdoderma lineare Schmidle \& Lauterborn & & & 72 & & & \\
\hline \multicolumn{7}{|l|}{ Chlorophyceae } \\
\hline Chlorella vulgaris Kessler \& Huss & & 36 & & & & \\
\hline Desmodesmus quadricauda (Turpin) Brébrisson & & 2238 & 8013 & & 2888 & 830 \\
\hline Dictyosphaerium pulchellum Wood & & & 36 & & & \\
\hline Eutetramorus fotti (Hind.) Kom. & & & & & & 36 \\
\hline Oocystis sp. Lemmermann & & & & 4 & & 36 \\
\hline Pediastrum duplex Meyen & & & 36 & & & \\
\hline Scenedesmus acuminatus (Lagerheim) Chodat & & 18 & 36 & & & \\
\hline \multicolumn{7}{|l|}{ Zygnematophyceae } \\
\hline Desmidium sp. (C.A. Agardh) & & & & & & 36 \\
\hline \multicolumn{7}{|l|}{ Euglenophycea } \\
\hline Euglena sp. Ehrenberg & & & 36 & & & \\
\hline Lepocinclis sp. Perti & & 36 & & & & \\
\hline Phacus sp. Pochmann & & & & & & 36 \\
\hline \multicolumn{7}{|l|}{ Bacillariophyceae } \\
\hline Aulacoseira granulata (Ehrenberg) Simonsen & & & 36 & & 36 & \\
\hline Cyclotella sp. (Kützing) Brébisson & & 126 & & & & \\
\hline Fragilaria sp. Lyngbye & & 343 & 144 & & 938 & 433 \\
\hline Navicula sp. Bory de Saint-Vincent & & & & 4 & & 36 \\
\hline Nitzschia sp. & & & 36 & & & \\
\hline Pinnularia sp. Ehrenberg & & & & & 36 & 36 \\
\hline Surirella sp. Turpin & & & & & 36 & 72 \\
\hline Tabellaria sp. Kützing & & 18 & & & & \\
\hline $\begin{array}{l}\text { Urosolenia cf. longiseta (Zacharias) Edlund et } \\
\text { Stoermer }\end{array}$ & & & & & 108 & \\
\hline \multicolumn{7}{|l|}{ Cryptophyceae } \\
\hline Cryptomonas sp. Ehrenberg & & 18 & & & 36 & \\
\hline Densidade total & 14 & 3176 & 8915 & 7 & 4404 & 1552 \\
\hline
\end{tabular}


Em abril registrou-se uma maior riqueza e também maior densidade de Cyanophyceae, principalmente daquelas filamentosas. $O$ oposto foi verificado para a Classe Bacillariophyceae, que registrou os maiores valores na amostragem realizada em agosto de 2013.

Chlorophyceae foi mais abundante em agosto de 2013 no ponto P3. Esse mês é caracterizado como período seco o que pode ter favorecido o desenvolvimento das algas pertencentes às clorofíceas. Resultado semelhante já foi observado nos reservatórios de Paranoá (BRANCO; SENNA, 1996), de Guarapiranga (BEYRUTH, 2000) e de Barra Bonita (CALIJURI et al., 2002). Nos dois últimos reservatórios, ambos no Estado de São Paulo, os estudos mostraram maior abundância de Chlorophyceae associada ao período de circulação da água, elevada transparência e concentrações mais altas de nutrientes, particularmente nitrato e fosfato.

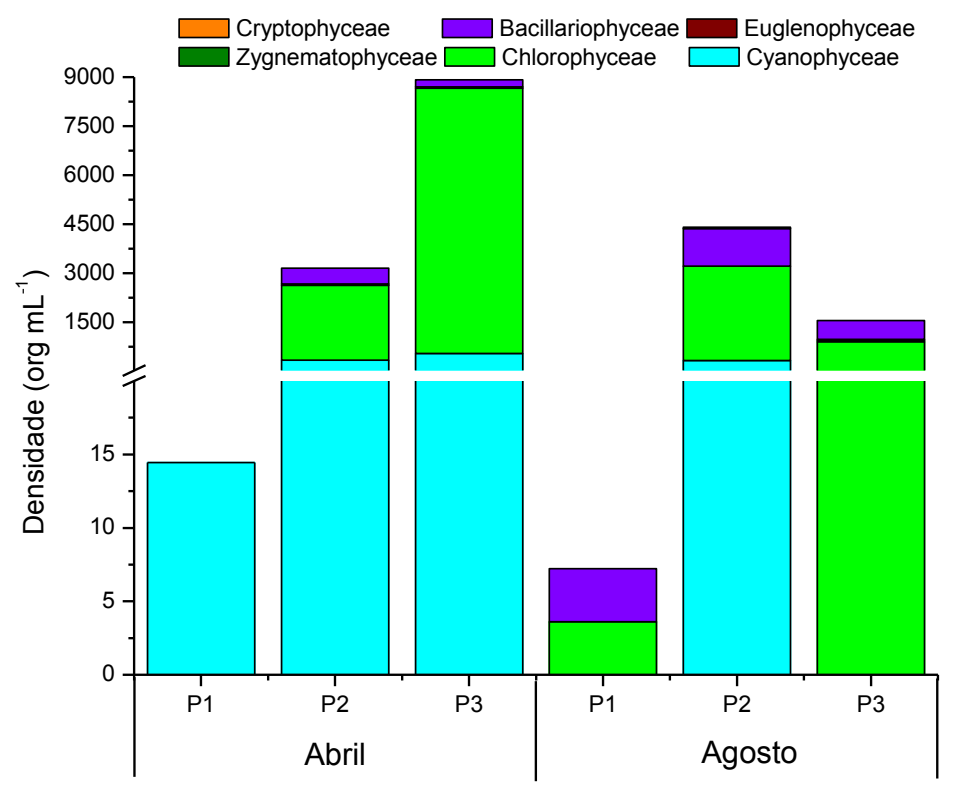

Figura 3. Densidade (org $\mathrm{mL}^{-1}$ ) das classes fitoplanctônicas registradas nos pontos de coleta no Parque do Bicão nos meses de abril e agosto de 2013.

As 30 espécies fitoplanctônicas foram agrupadas em 12 grupos funcionais (Tabela 4). Destes, quatro foram formados por Cyanophyceae (K, Lo, S1 e M) e Bacillariophyceae $(P, A, D, N)$; três por Chlorophyceae $(X 1, F$ e J), um por Zygnematophyceae $(N)$, Euglenophyceae (W1) e Cryptophyceae (Y). A grande maioria destes grupos é típica de 
ambientes mesotróficos. A única exceção foi a associação $\mathrm{J}$ que indica condição eutrófica (REYNOLDS et al., 2002).

No presente estudo, o grupo funcional $\mathrm{J}$ foi representado principalmente por $D$. quadricauda. Essa espécie ocorreu em altas densidades no período de seca também no trabalho de Gentil et al. (2008), esses autores correlacionaram esse resultado à maior disponibilidade de nutrientes provenientes da ressuspensão do fundo, aos valores mais baixos de $\mathrm{pH}$ e aos maiores valores de transparência.

Tabela 4: Contribuição relativa (\%) baseada na densidade dos grupos funcionais do fitoplâncton (GF) registrada nos pontos de coleta no Parque do Bicão nos meses de abril e agosto de 2013.

\begin{tabular}{|c|c|c|c|c|}
\hline \multirow{2}{*}{ Táxon } & \multirow{2}{*}{ GF } & \multicolumn{3}{|c|}{ Ocorrência (\%) } \\
\hline & & P1 & P2 & P3 \\
\hline $\begin{array}{l}\text { Aphanocapsa delicatissima, Aphanocapsa } \\
\text { koordersii, Epigloeosphaeria brasilica }\end{array}$ & K & & & \\
\hline $\begin{array}{l}\text { Merismopedia glauca, Rhabdoderma } \\
\text { lineare }\end{array}$ & Lo & 50,0 & 5,0 & 1,6 \\
\hline $\begin{array}{l}\text { Limnothrix sp., Planktolynbgya limnetuca, } \\
\text { Pseudanabaena galeata }\end{array}$ & S1 & 0,0 & 0,0 & 0,6 \\
\hline Raphidiopsis curvata & M & 0,0 & 2,7 & 0,0 \\
\hline Chlorella vulgaris & $\mathrm{X} 1$ & 0,0 & 0,6 & 0,0 \\
\hline $\begin{array}{l}\text { Desmodesmus quadricauda, Pediastrum } \\
\text { duplex, Scenedesmus acuminatus }\end{array}$ & J & 0,0 & 68,3 & 72,1 \\
\hline $\begin{array}{l}\text { Dictyosphaerium pulchellum, } \\
\text { Eutetramorus fotti, Oocystis sp. }\end{array}$ & $\mathrm{F}$ & 25,0 & 0,0 & 2,5 \\
\hline Desmidium sp., Tabellaria sp. & $\mathrm{N}$ & 0,0 & 0,3 & 1,2 \\
\hline Euglena sp., Lepocinclis sp., Phacus sp. & W1 & 0,0 & 0,6 & 1,4 \\
\hline $\begin{array}{l}\text { Aulacoseira granulata, Fragilaria sp., } \\
\text { Navicula sp., Pinnularia sp., Surirella sp. }\end{array}$ & $P$ & 25,0 & 17,3 & 19,6 \\
\hline Cyclotella sp., Urosolenia cf. longiseta & A & 0,0 & 3,2 & 0,0 \\
\hline Nitzschia sp. & D & 0,0 & 0,0 & 0,2 \\
\hline Cryptomonas sp. & Y & 0,0 & 0,7 & 0,0 \\
\hline
\end{tabular}

O grupo funcional $F$ apresentou grande representatividade na comunidade fitoplanctônica apenas na amostragem em agosto de 2013. Esse resultado provavelmente se relaciona às condições de menor intensidade de chuva no período seco, haja vista que a associação $\mathrm{F}$ tem afinidade ambiental por epilímnio claro (REYNOLDS et al., 2002). 


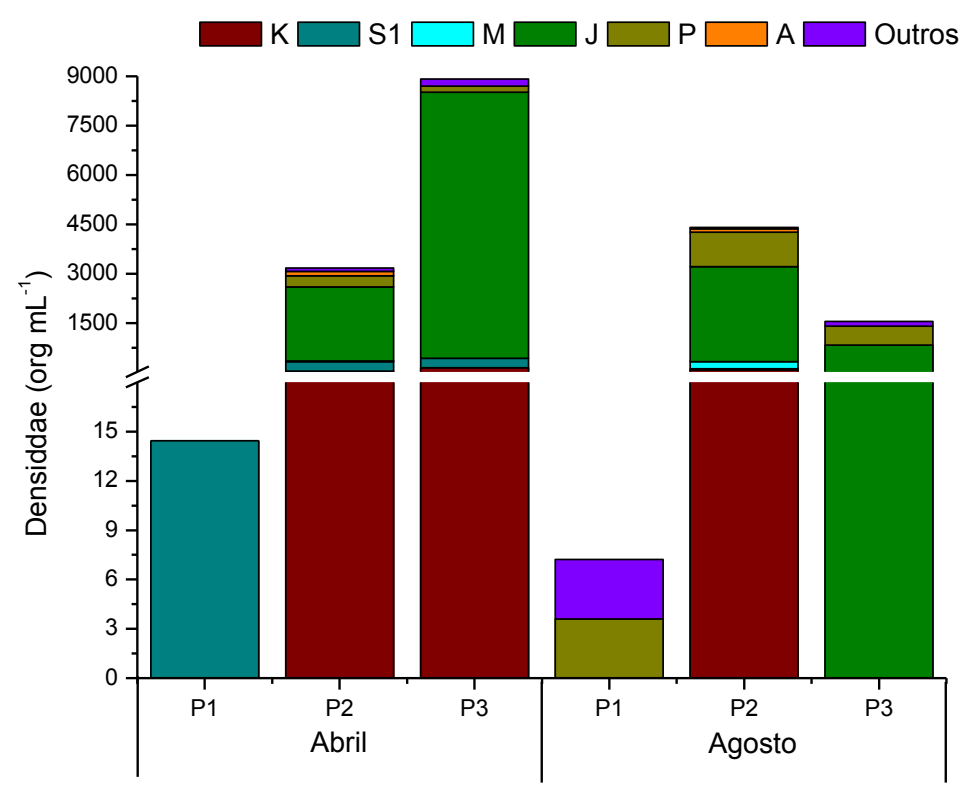

Figura 4. Densidade (org $\mathrm{mL}^{-1}$ ) dos principais grupos funcionais do fitoplâncton registrada nos pontos de coleta no Parque do Bicão nos meses de abril e agosto de 2013.

Apesar do maior número de espécies em abril de 2013, os maiores índices de diversidade foram registrados em agosto de 2013. Isso ocorre, pois o índice de ShannonWiener leva em consideração também a equitabilidade da densidade dessas espécies nos pontos de amostragem (ODUM, 1988).

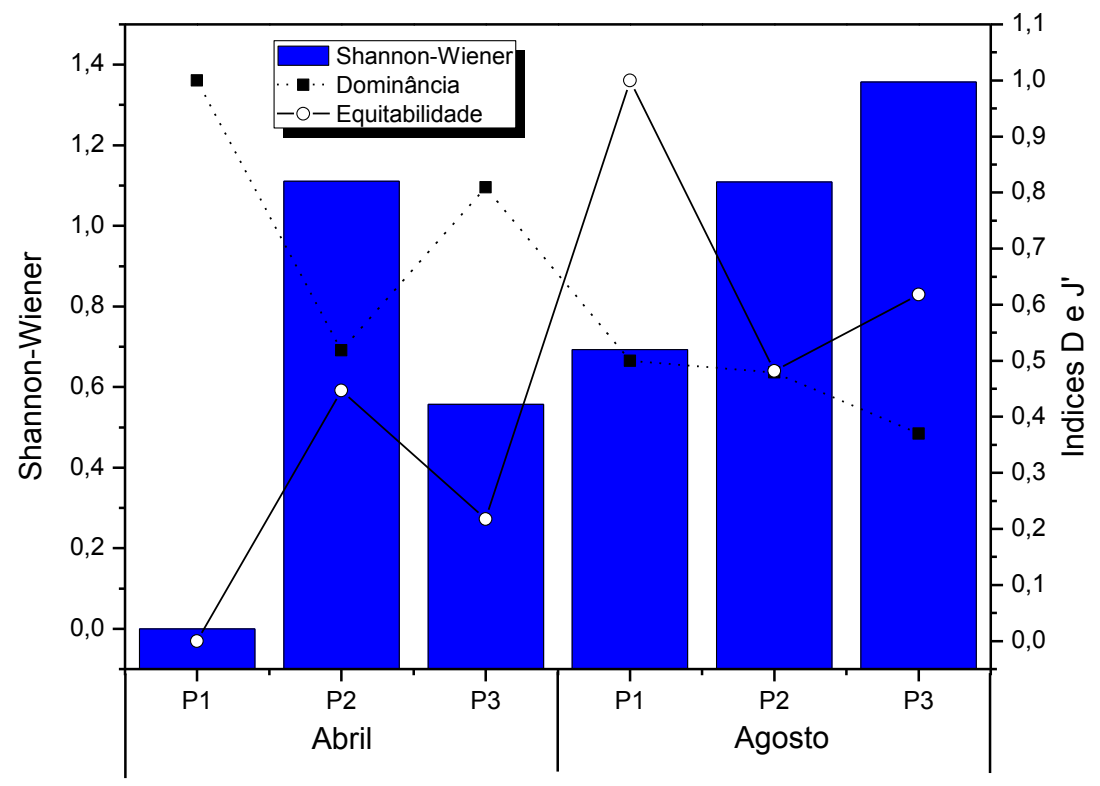


Figura 5. Índices de diversidade (Shannon-Wienner), dominância (D) e equitabilidade (J') da comunidade fitoplanctônica registradas nos pontos de coleta no Parque do Bicão nos meses de abril e agosto de 2013.

\section{CONCLUSÕES}

A análise da comunidade fitoplanctônica do Parque do Bicão revelou uma riqueza expressiva de espécies, típico de sistemas tropicais. As clorofíceas, cianobactérias e diatomáceas foram componentes importantes desta comunidade.

A abordagem dos grupos funcionais do fitoplâncton constituiu uma ferramenta útil para a compreensão da comunidade fitoplanctônica em sistemas urbanos, apesar de ter sido originalmente formulado para sistemas temperados.

Portanto, a região onde se localiza o Parque do Bicão, pela expressiva diversidade de algas, deveria ser considerada para a utilização na preservação do equilíbrio natural e proteção das comunidades aquáticas. Contudo, isso só será possível quando as medidas que visem a sua recuperação e preservação sejam efetivamente implantadas.

\section{REFERÊNCIAS}

BEYRUTH, Z. Periodic disturbances, trophic gradient and phytoplankton characteristics related to cyanobacterial growth in Guarapiranga Reservoir, São Paulo State, Brazil. Hydrobiologia, vol. 424, p. 51-65, 2000.

BICUDO C. E. M.; MENEZES M. Gêneros de Algas de Águas Continentais do Brasil: chave para identificação e descrições. São Paulo: Rima; 2005.

BORGES, P. A. F.; TRAIN, S.; RODRIGUES, L. C. Estrutura do fitoplâncton, em curto período de tempo, em um braço do reservatório de Rosana (ribeirão do Corvo, Paraná, Brasil). Acta Scientiarum, v.30, p. 57-65, 2008.

BRANCO, C. W. C.; SENNA, P. A. C. Phytoplankton composition, community structure and seasonal changes in a tropical reservoir (Paranoá Reservoir, Brazil). Algological Studies, v. 81, p. 69-84, 1996. 
CALIJURI, M. C.; SANTOS, A. C. A.; JATI, S. Temporal changes in the phytoplankton community structure in tropical and eutrophic reservoir (Barra Bonita, SP, Brazil). Journal of Plankton Research, v. 24, p. 617-634, 2002

CROSSETI, L. O.; BICUDO, C. E. M. Effects of nutrient impoverishment on phytoplankton biomass: a mesocosms experimental approach in a shallow eutrophic reservoir (Garças Pond), São Paulo, southeast Brazil. Rev. bras. Bot.,v. 28, n.1, p. 95-198, 2005.

CUNHA, D. G. F.; CALIJURI, M. C. Variação sazonal dos grupos funcionais fitoplanctônicos em braços de um reservatório tropical de usos múltiplos no estado de São Paulo (Brasil). Acta Botanica Brasilica, v.25, n.4, p. 822-831, 2011.

DANTAS, E. W.; MOURA, A. N.; BITTENCOURT-OLIVEIRA, M. C.; ARRUDA-NETO, J. D. T.; CAVALCANTI, A. D. C. Temporal variation of the phytoplankton community at short sampling intervals in the Mundau reservoir, Northeastern Brazil. Acta Botanica Brasilica, v. 22, p. 970-982, 2008.

DAJOZ, R. 1978. Ecologia geral. $3^{\circ}$ Ed. Editora Vozes LTDA: Petrópolis. 472 p.

ESPÍNDOLA, E. L. G. Dinâmica da associação congenérica das espécies de Notodiaptomus (Copepoda, Calanoida) no reservatório de Barra Bonita, SP. 1994. 363 f. Tese (Doutorado em Ciências da Engenharia Ambiental) - Centro de Recursos Hídricos e Ecologia Aplicada, Escola de Engenharia de São Carlos, Universidade de São Paulo, São Carlos, 1994.

HAMMER, U. T.; HARPER, D.; RYAN, P. PAST: Paleontological statistics software package for education and data analysis. Paleontological Electronic, v. 4, n. 1, 9 p. 2001

HUSZAR, V. L. M.; CARACO, N. The relationship between phytoplankton composition and physical-chemical variables: a comparison of taxonomic and morphological-functional descriptors in six temperate lakes. Freshwater Biology, v. 40, n. 4, p. 679-696, 1998. 
HUSZAR, V., KRUK, C.; Caraco, N. Steady state of phytoplankton assemblage of phytoplankton in four temperate lakes (NE USA). Hydrobiologia, v. 502, p. 97-109, 2003

HUTCHINSON, G. E. The paradox of plankton. American Naturalist, v.95,p. 137-145, 1961.

GENTIL, C. R.; TUCCI, A.; SANT'ANNA, C. L. Dinâmica da comunidade fitoplanctônica e aspectos sanitários de um lago urbano eutrófico, São Paulo, SP. Hoehnea, v. 35, n. 2, p. 265-280, 2008.

LONGHI, M. L.; BEISNER, B. E. Environmental factors controlling the vertical distribution of phytoplankton in lakes. Journal of Plankton Research, v. 31, p. 1195-1207, 2009.

MARGALEF, R. 1983. Limnologia. Omega, Barcelona.

ODUM, E. P. Ecologia. Rio de Janeiro: Guanabara Koogan, 1988. 434 p.

REYNOLDS, C. S.. Phytoplankton periodicity: the interations of form, function and environmental variability. Freshwater Biology, v. 14, p. 111-142, 1984.

REYNOLDS, C. S. Functional morphology and the adaptive strategies of freshwater phytoplankton. In: SANDGREN, C. D. (ed.). Growth and reproductive strategies of freshwater phytoplankton. Cambridge University Press, Cambridge, 1988, p. 388-433.

REYNOLDS, C. S. The Ecology of Phytoplankton (Ecology, Biodiversity and Conservation). Cambridge University Press, Cambridge, 2006, 550 p.

REYNOLDS, C. S.; HUSZAR, V.; KRUK, C.; NASELLI-FLORES, L.; MELO, S. Towards a functional classication of the freshwater phytoplankton. Journal of Plankton Research, v. 24, p. 417-428, 2002.

ROS, J. Prática de ecologia. Barcelona:Omega,1979, 181 p. 
SANTOS, R. M. Estrutura das comunidades fitoplanctônica e zooplanctônica, com ênfase na produção secundária do zooplâncton, e fatores ambientais relacionados nos reservatórios do Baixo Rio Tietê, SP. 2010. 382 p. Tese (Mestrado em Ecologia e Recursos Naturais0. Universidade Federal de São Carlos, São Carlos, 2010

TARPANI, R. R. Z. Análise ambiental de Micro-Bacia hidrográfica visando a prevenção de danos ambientais e econômicos. 2008. 64 f. Trabalho de Conclusão de Curso (Graduação em Engenharia Ambiental), Escola de Engenharia de São Carlos, Universidade de São Paulo, São Carlos. 2008.

TUCCI, A.; SANT'ANNA, C. L.; GENTIL, R. C.; AZEVEDO, M. T. P. Fitoplâncton do lago das Garças, São Paulo, Brasil: um reservatório urbano eutrófico. Hoehnea, v. 33, n. 2, p. 147-175, 2006.

TUNDISI, J. G. Água no século XXI: enfrentando a escassez. São Carlos: RiMa, IIE, 248p, 2003.

UHELINGHER, V. Étude statistique des méthodes de dénobrement planctonique. Arch. Sci., v. 17, n. 2, p.121-123, 1964.

UTERMÖHL, H. Zur Vervolkomnung der quantitative Phytoplankton: Methodik Mitteilung Internationale Vereinigung Theoretische und Angewandte Limnologie, v. 9, p. 1-38, 1958. 